\title{
Can Survey Measures Predict Subsequent Key Performance Indicators of Maritime Safety? Confirmatory and Exploratory Analyses of the Association between Self-report and Objective Indices
}

\author{
Line Raknes Hjellvik¹ \& Bjørn Sætrevik,1
}

\begin{abstract}
We need reliable knowledge about what causes and prevents accidents in order to manage safety. Such associations are often investigated with self-report measures of psychological factors. However, few studies in the maritime industry have investigated the extent to which self-report measures predict objectively registered accidents. The current pre-registered study used structural equation modelling to test whether self-reported individual safety-related factors predicted objective safety outcomes in the following year. The study was conducted among crew on chemical tanker vessels operating in Arctic and Baltic waters. The pre-registered model and the expected associations between self-reported safety factors and safety outcomes were not supported. However, an exploratory model based on the pre-registered hypotheses supported an association between self-reported safe behaviour and the overall number of safety outcomes. While much safety research builds on the assumption that self-reported behaviour, attitude or cognitions are causally related to actual accidents, the current study shows that such a relationship can be difficult to confirm. We recommend more caution in assuming a causal relationship between self-reported psychological factors and safety outcomes until a causal relationship can be established.
\end{abstract}

\section{Keywords}

Chemical tanker vessels, maritime safety, self-report and objective outcomes, pre-registered study, structural equation modelling

\section{1: Introduction}

Safety research in the maritime industry has covered both technological and human factors in order to facilitate work environments that reduce the risk of accidents. Many studies have investigated non-technical skills in the shipping industry, but investigations of the relationship between behaviour and accidents or reports of incidents are lacking (Hetherington, Flin, \& Mearns, 2006). Measures that have been used to investigate human factors, errors and reliability relevant for safety includes e.g. the Human Factors Analysis and Classification System (Celik \& Cebi, 2009; Chauvin, Lardjane, Morel, Clostermann, \& Langard, 2013), human reliability analysis (Akyuz \& Celik, 2015) and surveys (Fenstad, Dahl, \& Kongsvik, 2016; Lu \& Tsai, 2010; Nævestad, Phillips, Størkersen, Laiou, \& Yannis, 2019; Sandhåland, Oltedal, Hystad, \& Eid, 2017). Surveys are a resource-efficient way of gathering the employees' perspectives, attitudes and experiences. It is thus possible to measure several variables simultaneously, which allows for a comprehensive analysis of the safety condition of an organization and the relationships between various variables assumed to be related to safety. However, all self-report measures have limitations that bias the results and limit the conclusions and causal inferences. The object of the current study is therefore to investigate if factors previously identified as safety critical (see Hjellvik, Aga, \& Sætrevik, 2019) have an impact on behaviour categorized as unwanted or dangerous in the same population where data were collected.

The current study was conducted among crew on chemical tanker vessels (CTVs) operating in Arctic and Baltic waters. Work on CTVs may be characterized as hazardous with a potential for large-scale accidents that could have severe consequences for both health and the environment (Arslan \& Er, 2008). Work aboard the vessels may be influenced by hydrodynamics, darkness and environmental conditions such as cold or warm weather. The crew are away from their homes for longer periods of time, and it may be difficult to separate working hours from leisure time. Multinational crews are common in the maritime industry, and this could impact interpersonal communication and collaboration. Further, the crew are responsible for various technical operations and monitoring the condition of various chemical liquids to ensure that no serious effects such as fire, corrosiveness, self-reaction and poisoning occur (Celik, 2010). Work on CTVs is therefore highly regulated and requires competence and knowledge beyond what is expected in other merchant fleets. Risky occupations have a safety management system (SMS) in order to monitor and react to how the safety level changes, and to enhance safety by improving the relevant aspects of the sociotechnical work environment. System design, work-environment, personnel readiness and interpersonal and organizational factors should be considered in order to maintain safety on maritime vessels (Chauvin, 2011; Ding, Han, Li, \& Deng, 2014).

\section{1: Individual Factors that may impact Safety}

1.1.1: Safety attitude and reporting attitude. An organization's safety culture comprises features such as values, safety perceptions, competences, behaviour and attitudes (Chauvin, 2011). Attitudes are the summarized product of affect and cognition, and attitude formation may be described as acceptance or rejection of an object (Crano \& Prislin, 2006). Both organizational and interpersonal factors may impact attitudes, and norms and incentives related to safety may be prevailing elements that contribute to individual beliefs about expected safety behaviour. Ajzen (1991) suggested that attitudes, subjective norms and perceived control predict behaviour intentions with high accuracy. Cox and Cox (1991)

\footnotetext{
${ }^{1}$ Operational Psychology Research Group, Faculty of Psychology, 
found that safety attitudes were determined by evaluations and beliefs related to arrangements for safety, such as rules and procedures, scepticism and responsibility and risk. The relationship between attitudes and behaviour is welldocumented (Donald \& Canter, 1994; Rundmo \& Hale, 2003), and attitude to safety has been indicated to be a relevant mediator in a causal chain of safety critical factors in survey research (Hjellvik et al., 2019). This indicates that the crew's thoughts and beliefs about risks and organizational structures impact their self-reported work performance. However, there remains a need to investigate whether attitudes predict actual behaviour.

Further, the attitudes that the crew on CTV have to the reporting system may influence their actual reporting behaviour. Reporting systems for incidents are often used in the maritime industry under the assumption that such monitoring may highlight various safety critical challenges such as system faults, human error, lack of system knowledge or excessive workload. Systematic reporting may facilitate preventive measures. However, the reliability of reporting in the maritime industry has been shown to be of various quality (Storgård, Erdogan, Lappalainen, \& Tapaninen, 2012). Whether a given incident is reported depends on the employees' willingness to invest time and energy in writing accurate reports when an incident occurs. It is thus reasonable that the employee's feelings and thoughts about these systems influence the frequency and quality of reports. Feedback and commitment from the management, repercussions and uncertainty about what incidents to report are some of the factors that may impact reporting motivation (see e.g. Pfeiffer, Manser, \& Wehner, 2010 for a proposition of antecedents). It is thus necessary to investigate whether psychological factors such as the crew's safety and reporting attitudes are associated with the crew's actual safety and reporting behaviour.

1.1.2: Situation awareness. The crew's safe behaviour may also be determined by their ability to accurately detect and understand safety critical cues and anticipate further development of various scenarios that may follow given actions. The crew on CTVs have challenging work operations such as navigation, mooring, and handling and lifting of dangerous cargo. An operator needs to have an accurate online assessment of the current environment, where elements are discovered and assessed relative to other elements. This should facilitate a mental model where an operator has the necessary information to predict the potential outcomes of decisions, sometimes referred to as "situation awareness" (SA). Endsley (1995b) described SA as a tripartite information process, where perception, understanding and prediction of a dynamic situation were represented by the term. For crew on CTVs, perception of critical cues such as warning signals for the status of the content of chemical cargo are vital for further decisions of handling such cargo. This requires that the operator has sufficient attention and knowledge about the system and that the work environment provides the operator with information that is suitable for the task and the operator's training and capacities. Accurate perception of relevant safety cues, and knowledge about the operative work environment, should provide a better basis for successful decisions, and lead to fewer dangerous incidents. Maintaining an accurate
SA may be challenging as there are numerous factors that could impact an operator's ability to identify elements, develop a coherent understanding of the relationship between them as well as anticipate the products of such outcomes. Limited attention, excessive workload and little experience with the current system are some of the factors that may reduce an operator's ability to acquire the SA that is necessary for safe performance.

Various measurement approaches have been used to assess SA (Salmon, Stanton, Walker, \& Green, 2006), and SA has been found to predict performance and decision making (Endsley, 2019; Strybel, Vu, Kraft, \& Minakata, 2008). The concept of SA has been subject to debate (Dekker, Hummerdal, \& Smith, 2010), and Dekker and Hollnagel (2004) suggested a shift from the focus on cognition to a focus on the products of cognition. SA is often evaluated in terms of how well an individual's information processing related to a given task complies with some predetermined criteria (e.g. the Situation Awareness Global Assessment Technique, SAGAT, Endsley, 1995a). Such methods can be useful to get an immediate and reliable assessment of an operator's SA which may provide detailed information about perception and cognition that could facilitate further improvements of sociotechnical systems. However, the work-environment aboard CTVs comprises a range of various operations and collaboration. It would thus be resource extensive and unrealistic to measure the SA of all crew members across all their tasks. Previous studies have developed and supported an approach measuring the selfreported experience of SA across work settings (Hjellvik et al., 2019; Sætrevik, 2013; Sætrevik \& Hystad, 2017, 2019). Measuring the crew's SA, as well as other safety factors "in the field" aboard CTVs provides information about the safety level aboard the vessels and the anticipated products of the crew's self-reported cognitive states and performance. Survey methods allow comparison between organizations, and several measurements may be used to compare levels of safety within the same organization. The current study adopts similar measures, but also tests whether self-reported SA measures predict incidents in the year following the selfreport. It is reasonable to expect that crew who state that they have accurate SA will have the ability to work safely and thus prevent unwanted incidents.

1.1.3: Safe behavior. Safe behaviour on CTVs is expected to be determined by the crew's motivation and ability to comply with the safety management system. The literature on maritime safety often highlight that accidents are caused by human error, although various perspectives on failure in complex systems have been discussed (Dekker \& Nyce, 2012). The cause of an incident may differ depending on perspective (Rasmussen, 2003), and recognizing factors that may prevent hazardous behaviour is considered to be paramount for safety. Self-report of safe or unsafe behaviour is often used as a proxy for actual behaviour (e.g. Hjellvik et al., 2019; Nævestad et al., 2019; Sneddon, Mearns, \& Flin, 2013; Sætrevik \& Hystad, 2017, 2019). The current study used self-report to measure the personal and collective safety responsibilities of the crew working on CTVs. This builds on the assumption that the crew are honest when they are to report their experience of their own and others' safety behaviour aboard the vessels. It also relies on the crew being 
able to recognize unsafe acts when they are committed or observed, and to correctly recall them in the survey. Individuals who state that they do not exhibit risk behaviour should contribute to a safer work environment, and these subjective statements should thus also be reflected in objective records of behaviour.

1.1.4: Incidents and "near-miss" as safety outcomes. As the aim of most safety research is to predict and control the consequences of accidents, the final objective is to identify factors that may predict observed accidents. Hofmann and Stetzer (1996) found that survey measures of safety climate and unsafe behaviour were associated with chemical processing plant accidents in the three preceding years. Although most industries have a system for monitoring and recording accidents, gathering and using accident rates as an outcome measure is time consuming, requires close collaboration with an industry partner, and may be difficult to interpret. The passing of time, technological and organizational developments may lead the industry partners to be less interested in the causes of past accidents (Zohar, 2000), which may decrease the motivation for performing this kind of research.

In high-reliability organizations, major accidents are rare, even when measured over time and across large organizations. It could thus be beneficial to use measures of incidents that have a more frequent occurrence (Zohar, 2000). We will use a ship-owning company's registration of key performance indicators (KPI) of unwanted incidents as objective outcome measures, as these are expected to have a relatively high frequency of occurrence. The first safety outcome is the recording of unwanted events or acts that have been reported and categorized as incidents that had the potential to trigger accidents or was categorized as an actual accident. The second safety outcome is the recording of "Near misses". These are the events that have passed through one or more of the safety-barriers but were terminated in time to prevent an accident (Hopkins, 2009). These reports are expected to be an indicator of the general safety level and may be the precursor of large-scale accidents.

\section{2: Research needs and Current study}

A previous analysis of self-reported variables in the current dataset (Hjellvik et al., 2019) has shown that organizational factors and safety attitudes predict safety behaviour. The self-reported statements about attitudes, cognition and safety policies were used as indicators for the crew's self-reported safety behaviour. However, self-report of behaviour has been shown to deviate from objective measures of the behaviour (Prince et al., 2008). Self-report relies on accurate perception, introspection and recall, but its accuracy is difficult to assess without additional measures. Social desirability is a common error source in survey research (see e.g. van de Mortel, 2008 for a review), and may be described as the tendency that participants have to portray themselves in a more favourable light by exaggerating behaviour that are considered advantageous and lessen negative attributes (Edwards, 1953; Pedregon, Farley, Davis, Wood, \& Clark, 2012). It is thus necessary to investigate if self-reported attitudes and behaviour predict objective safety outcomes. The current study builds on the previous mentioned study (Hjellvik et al., 2019). We will use individual self-report of attitudes and behaviour as predictors of a shipowing company's recorded safety outcomes in the twelve months following the survey data collection. Individual factors such as attitudes, situation awareness and behaviour (that were the mediators and outcomes from the previous study) will be tested against indices of actual recorded incidents. The respondents in this study were employees in a Norwegian company that owns and manages CTVs operating in Arctic and Baltic waters. While safety research is often concerned with identifying risk factors for accidents, the current study employs a preventive perspective where we seek to identify protective psychological capacities that may increase the safety level on CTVs.

1.2.1: Hypotheses and pre-registration. The overview of safety research above indicates that the crew's cognitions, attitudes and behaviour may be associated with actual safety outcomes. Positive attitudes to safety may indicate that the crew are motivated and able to maintain safety, and this could lead to proactive work ethics which reduce risk behaviour. From this, one would expect safety attitudes to be negatively associated with unwanted incidents that is registered by the safety management system ("Number of incidents"). Similarly, positive attitudes may lead to improved safety and thus reduce the number of unsafe situations that the crew identify and report ("Number of reports"). However, an overly positive attitude to safety may also indicate that the crew are complacent to safety issues, which may lead to an increase in the number of reports of incidents primarily filed by the crew ("Number of reports"). The associations to "Number of reports" are hence non-directional. This leads to the following expectations: "Safety attitude" will be positively associated with "Safe behaviour" (H1 a), "Safety attitude" will be negatively associated with "Number of incidents" (H1b) and "Safety attitude" will be associated with "Number of reports" (H1c).

It is further expected that individuals who perceive, understand and predict their work environment accurately will have both the confidence and ability to perform work safely. This leads to H2: "Situation awareness" will be negatively associated with "Number of incidents". Further, crew members who have positive attitudes to reporting should show complacent reporting behaviour. This would be reflected in a positive relationship between reporting attitudes and the number of reports filed. However, there may also be fewer incidents to report as a reliable reporting system may facilitate preventive measures and reduce unwanted incidents. This could result in a negative relationship between reporting attitudes and the number of reports filed. From this we will test whether there is an association between "Reporting attitude" and "Number of reports" (H3), while testing for both positive and negative relationships (non-directional hypothesis).

Safety research often use self-reported behaviour as an outcome variable, but it is necessary to investigate whether self-reported behaviour is reliably associated with actual behaviour. We therefore suggest H4: "Safety behaviour" will be negatively associated with "Number of incidents".

It is also reasonable to believe that the number of events will correlate with the number of "near miss", in that a higher number of "near miss" indicates a reduced safety level, which should also lead to a higher number of incidents. 
However, the ambiguity in the concept of reporting may also lead to the hypothesis that a higher number of reports represents complacent reporting behaviour and should thus be negatively associated with incidents. The final hypothesis is thus H5: "Number of incidents" will be associated with "Number of reports" (non-directional).

The study was pre-registered on the Open Science Framework (https://osf.io/cj8fr) in order to contribute to transparent research practices and the necessary research standards for reproducibility and reduction of questionable research practices. Pre-registration of studies contributes to a focus on theory development rather than significant results, and a sound theory specified a priori increases confidence in results independent of outcome (Van 'T Veer \& GinerSorolla, 2016). The research design with the hypotheses and a complete analysis plan was pre-registered after the collection of survey data, but before collection of safety outcomes (the results will indicate which relationships between survey variables that had already been tested). The confirmatory analyses follow the pre-registration, and further exploratory analyses are based on the pre-registered hypotheses. The pre-registered theoretical model is depicted in Figure 1 below.

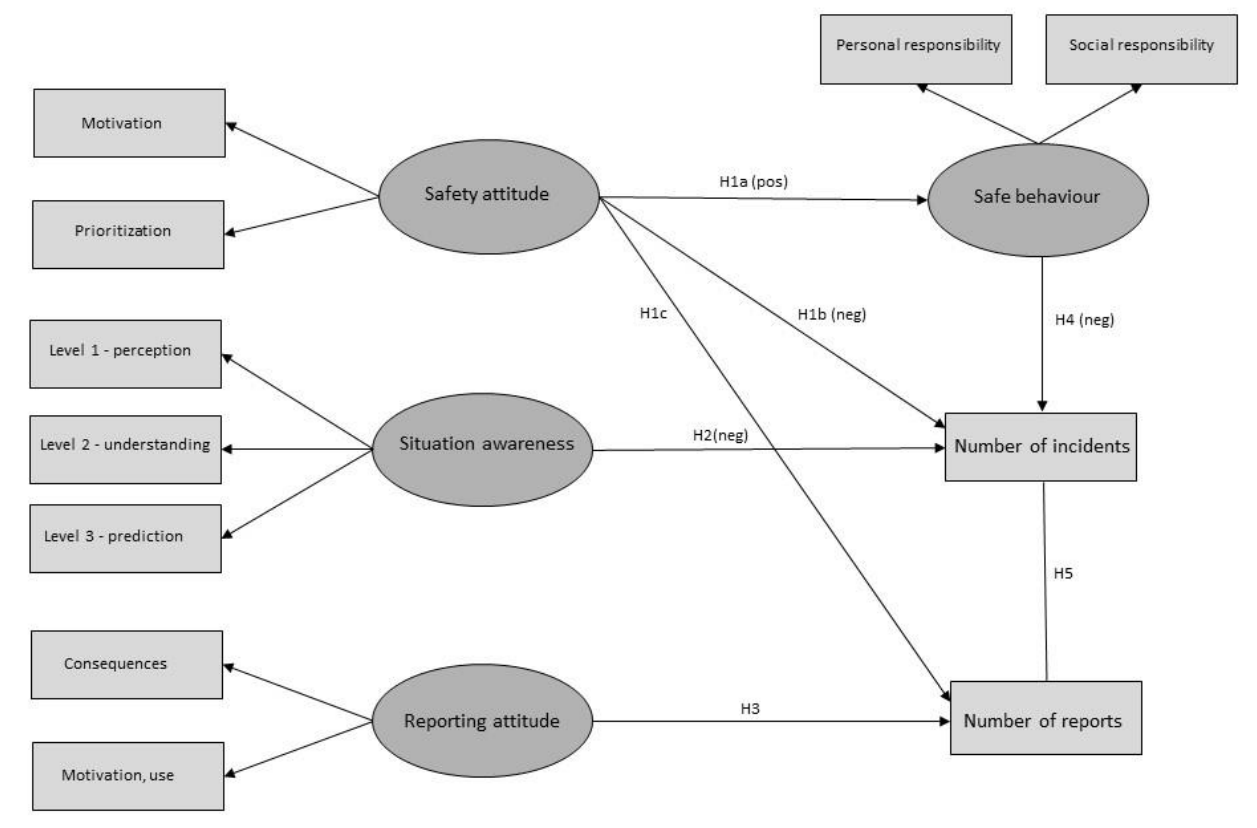

Figure 1: Theoretical model of the pre-registered hypotheses: H1-H5. In order to more fully explore the results, the current model was further developed into a model where "Number of incidents" and "Number of reports" were combined

\section{2: Methods}

\section{1: Measurement}

2.1.1: Procedure. A pen-and-paper survey was sent to all employees working on CTVs in a Norwegian ship-owning company. The company transports chemical cargo in the Arctic and Baltic waters. The surveys were coded to identify responses from the same vessel, and to be able to associate them with subsequent safety outcomes. The ship-owning company's HSEQ department distributed the surveys to the vessels. The ship-owning company held the code key to associate vessel name with survey code, but the ship-owning company did not receive the completed surveys or dataset. This system ensured that the researchers could not know the name of vessels or their crew, and the ship-owning company could not know the responses of crew members or specific vessels. The manning on the vessels is organized in shifts, and surveys were distributed to approximately 450 employees. The participants did not receive payment for participation, but they were encouraged to participate, and received one reminder to complete the survey. The survey had 122 statements about organizational, interpersonal and individual safety factors. The statements asked for demography and the extent to which the participants agreed to safety issues such as accuracy of situation awareness, the captain's and shipowning company's ability and willingness to focus on safety, their own safety behaviour and use of safety tools, and the extent to which they express positive safety attitudes. The statements were rated on a 5-point Likert scale from "Completely disagree" (1) to "Completely agree" (5). Some of the items were semantically reversed so that a score on "Completely agree" would count against the calculated variable. An overview of all items used in the survey is available online (https://osf.io/sfpu $5 /$ ), showing reversed items and how they assemble to variables.

The data for the outcome variables were registered and collected in the 12 months subsequent to the completed collection of survey data. The ship-owning company's safety management system registered the number of reports and incidents that occurred on the CTVs from April 2018 to April 2019. A total of 1540 reports were registered during this period, which corresponds to 4.2 unwanted events per day across the eighteen vessels. The data was registered for each vessel code and sent to the researchers to be added to the survey data set. Each crew member was assigned the number of incidents and "near miss" registered to their vessel. To 
compensate for the reduced variability in the dataset that this approach led to, the analysis took the clustering by vessel into account.

2.1.2: Participants. 328 surveys were returned to the researchers (73\% of the distributed surveys). Fifteen surveys were removed from the sample due to apparent language issues (e.g. ignoring all reversed items) and blank or identical answers on more than $33 \%$ of the items. In accordance with the pre-registered analysis, 21 captains were also excluded, resulting in a sample of 292 participants.

The participants worked on deck (59\%), in the engine room (31\%) and in the galley $(10 \%)$. There were $11-$ 28 participants from each of the eighteen vessels, all male. The participants were primarily from the Philippines $(75 \%)$ and Latvia (17\%), but participants from Russia, Norway, Lithuania and "Other" were also represented. The majority stated that they had worked for the ship-owning company for five years or more $(60 \%)$, but $49 \%$ stated that they had worked on the current vessel for less than a year. This indicates that the crew alternate between working on various vessels in the company's fleet. The first page of the survey provided consent information, and participants were informed that the survey was voluntary and anonymous. The project was submitted to The Norwegian Centre for Research Data (project 56912).

2.1.3: Variables. "Situation awareness" was measured with 13 statements that describe the perception, comprehension, and assessment of a situation. The items were developed by Sætrevik (2013) and represent Endsley's theoretical model (1995b) where SA has a tripartite division. Items thus represent the extent to which the participant tends to perceive safety-critical cues in their working-situation (level 1), understand contingencies between them (level 2) and predict how safety aspects of the situation will develop over time (level 3). The three levels are sub-levels of SA and level 1 was measured with four items, level 2 was measured with five items and level 3 was measured with four items. A sample item is: "I notice when an unsafe situation is about to arise at my workplace". Higher score on the variable indicates that the participant has accurate SA across various relevant worksettings. Five of the items were semantically reversed in order to prevent response biases. Cronbach's alpha for the scale was .81 .

"Safety attitude" was measured with 13 statements that describe the beliefs, feelings and thoughts the participants may have about safety. The items represent the participant's motivation to follow safety procedures and how they prioritize safety in their day-to-day work. The two subcategories "motivation" and "prioritization" were measured with nine and four items, respectively. Some of the statements were developed by the researchers and subject matter experts, some of the items were inspired by the shipowning company's safety campaign and some of the items were from scales developed by Nielsen and colleagues (2013) and Rundmo (1994). An example of a negatively phrased item is: "Safety procedures often stand in the way of getting the job done efficiently". Higher score on the variable indicates that the participant has a positive attitude to safety aboard the vessels. Four items were semantically reversed. Cronbach's alpha was 7 .

"Reporting attitude" was measured with 12 statements that describe the beliefs, feelings and thoughts the participants may have about the company's reporting system for incidents and "near-misses". The scale comprises items related to reporting motivation, use and consequences. The subcategories "consequences" and "motivation and use" were measured by eight and three items, respectively. Some of the items were inspired by Probst and Graso (2013) and were supplemented by additional items developed through iterative biennial surveys for a different maritime sector (see pre-registration Sætrevik \& Hjellvik, 2017), and through discussions with HSEQ officers for the current industry partner. A higher score on the variable indicates that the participant has positive attitudes to reporting unwanted incidents. Ten items were semantically reversed, and an example of a negatively phrased item is: "Reporting all accidents/near-misses will not be belpful to increase safety". Cronbach's alpha was .88.

"Safe behaviour" was measured with seven statements that describe actions with a positive or negative impact on safety that the participants may perform during their work. Some of the items were from the "Brief safety climate inventory" (Nielsen et al., 2013.) and supplemented with items developed in collaboration with industry experts to suit the current setting. Four of the items describe safety actions with relevance for how the respondent relates to other crew members (social responsibilities), while three of the items describe how the respondent's own actions are relevant for safety (personal responsibilities). Higher score on the variable indicates that the participant follows the safety procedures and expectations in their work. Five of the items were semantically reversed. An example of a negatively phrased item is: "I sometimes expose myself or others to danger in order to get the job done". Cronbach's alpha was .7.

"Safety KPIs" were recorded by the ship-owning company as part of their ordinary system for monitoring the vessels' safety through crew reports of unwanted incidents and "near-misses". All reports from the vessels were collected in the year after the surveys had been returned to the researchers. Each report was classified by the company as either constituting an actual incident or a "near-miss". The number of reports classified as "near-miss" per vessel were used as the "Number of reports" variable for all crew members assigned to that vessel. The remaining reports for the vessel were used as the "Number of incidents" for the crew members on that vessel. While the pre-registration suggested an additional follow-up analysis where incidents were classified according to severity, examining the recorded KPIs and discussing it with our contacts in the ship-owning company revealed that this was not feasible.

\section{2: Analyses}

Structural equation modelling (SEM) was used to test the preregistered hypotheses of associations between subjective and objective factors (using the gsem module in STATA). The pre-registered confirmatory structural model for testing of the hypotheses is shown in Figure 1. In addition, an exploratory model was tested after reviewing the results of the confirmatory model (shown in Figure 2).

\section{3: Results}

\section{1: Descriptive statistics}

All the predictor variables' average values were in the similar range, and indicating that the participants overall "agreed" with statements that their safety attitudes, cognitive abilities and behaviour are optimal for safe performance on 
the vessels (evaluated on scales from 1 to 5): "Safety attitude", $\mathrm{M}=3.99, \mathrm{SD}=0.48$, "SA", $\mathrm{M}=3.95, \mathrm{SD}=0.56$, "Reporting attitude", $\mathrm{M}=3.94, \mathrm{SD}=0.76$. and "Safe behaviour", $\mathrm{M}=$ $3.95, \mathrm{SD}=0.73$.

\section{2: Model testing}

The pre-registered structural model tested the associations between the crew's self-reported statements about safety and safety outcomes of their actual performance on the vessels. Results from testing the pre-registered model (see details in the online supplemental materials: https://osf.io/ks2xr/) did not support any of the tested hypotheses. However, an inspection and discussion with the industry partners revealed that the safety KPIs that were collected were defined differently than what was expected in the pre-registration. The separation between "Number of incidents" and "Number of reports" was more ambiguous than expected, which reduces our confidence that the pre- registered model is the best way to approach the research questions in the collected dataset. In order to more fully answer the pre-registered hypotheses, a follow-up exploratory structural model was constructed to test hypotheses $\mathrm{H} 1$ - H4 (thus excluding H5). The exploratory model is a simplification of the pre-registered model, where the two types of KPIs are collapsed (see Figure 2 below). The standardized root mean square residual (SRMR) was 0.058 for both the confirmatory factor analysis (CFA) and the SEM of the pre-registered model. For the exploratory model, the SRMR was 0.058 for the CFA and 0.056 for the SEM. These values are within the range of acceptable fit ( $\mathrm{Hu} \&$ Bentler, 1999). This indicates that the exploratory model provides a slightly better fit than the pre-registered model. The extent to which the hypotheses are supported in the exploratory model is reviewed below.

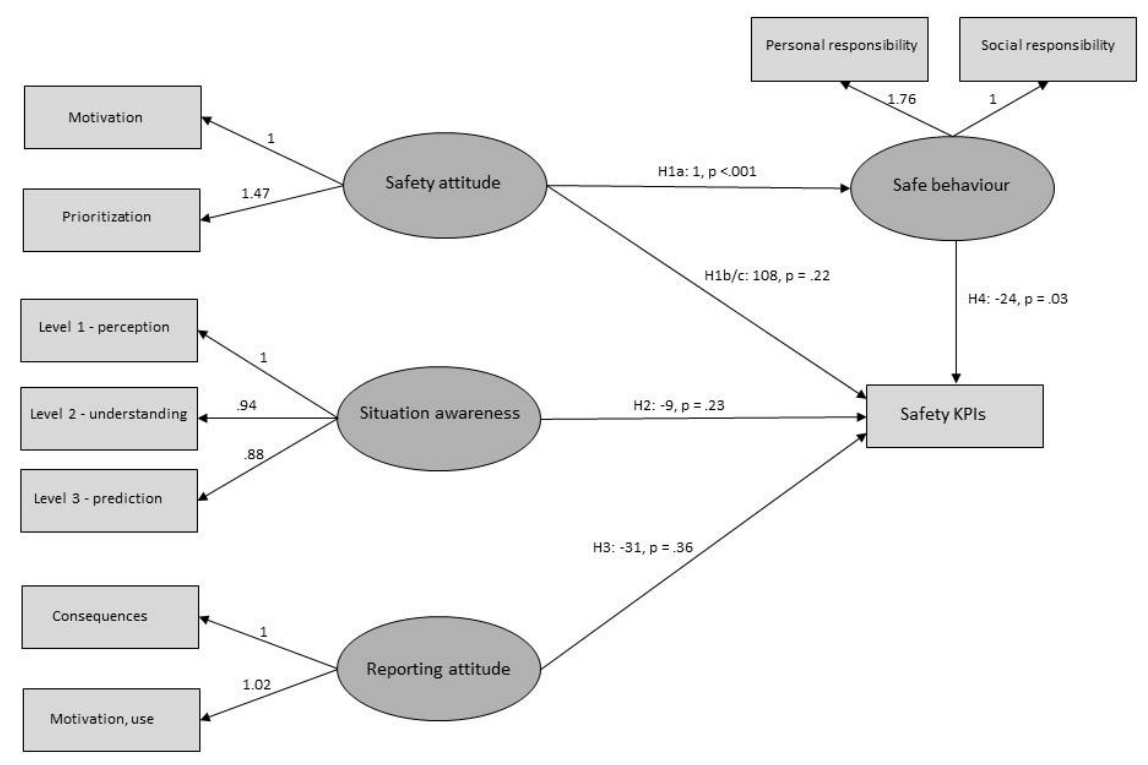

Figure 2: Results from the exploratory SEM-analysis where the total number of reported incidents are combined in one outcome variable. The figure shows unstandardized coefficients and two-tailed p-values, marked with bypothesis enumeration from the pre-registration. The pre-registration describes a combination of one-and two-tailed tests, but we have chosen to use two-tailed test for simplicity and rigor and the choice of test does not change rejection or support of the bypotheses. Unstandardized coefficients are chosen to make it easier to see the predicted change in KPIs. Error terms and observed indicators are not represented in the model presented above.

3.2.1: Support for H1. The positive association between "Safety attitude" and "Safe behaviour" (H1a) has previously been supported in the current dataset and was included in order to make the current models coherent. It is thus possible to compare this association with the remaining associations. As expected, H1a was supported in the exploratory model. The association indicates that those of the crew who have positive safety attitudes also perform their work safely.

The exploratory model did not show a significant association between "Safety attitude" and the total number of KPIs (H1b and c combined). This indicates that the beliefs the crew have about safety in their work did not impact the number of reports or incidents from their vessel.
3.2.2: Support for H2. "SA" was not significantly associated with the total number of KPIs in the exploratory model (H2'). This indicates that the accuracy of the crew's perception, comprehension and prediction of the safety aspects of their work did not impact the number of reports or incidents from their vessel.

3.2.3: Support for H3. "Reporting attitude" was not significantly associated with the total number of KPIs in the exploratory model $\left(\mathrm{H}^{\prime}\right)$. This indicates that the thoughts and feelings the crew have about the reporting system do not impact the number of reports or incidents from their vessel.

3.2.4: Support for H4. "Safe behaviour" was negatively associated with the total number of KPIs in the exploratory model (H4'). This indicates that there are fewer incidents on 
vessels with crew members that state that they follow the safety procedures in their work.

3.2.5: Support for H5. The relationship between "Number of incidents" and "Number of reports" was not tested in the exploratory model as the incidents and reports were combined into one variable.

\section{4: Discussion}

\section{1: Summary of results}

The literature has suggested that various individual factors influence safety-critical behaviour. These factors are typically measured by self-report but are assumed to impact the objective risk of accidents in the workplace. The present study aimed to test whether self-reported individual factors influence forthcoming objective safety outcomes. Data were collected among the crew employed in a ship-owning company that transports chemical cargo. The crew's selfreport of "Safe behaviour" was associated with the shipowning company's key performance indicators in the exploratory model, but the remaining hypotheses and the preregistered model were not supported.

4.2.1: Prediction of safety outcomes. As shown in a previous analysis of this dataset (Hjellvik et al., 2019), "Safety attitude" was associated with "Safe behaviour" (H1a). This indicates that crew who state that they have positive attitudes to safety also state that they follow procedures and regulations in their work. This association was included in the current analysis model in order to complete the model and allow comparison with the other associations. The remaining associations ( $\mathrm{H} 1 \mathrm{~b}$ - H5) were not supported in the pre-registered model. This indicates that we were not able to predict the independent effects of crew's evaluation of their attitudes, SA and behaviour aboard the vessels on their vessel's safety outcomes in the following year.

Due to uncertainty regarding how the company measured the KPIs and in order to better answer some of our pre-registered hypotheses, we additionally tested an exploratory model of the pre-registered hypotheses between survey responses and safety outcomes. Our initial and preregistered expectation of the ship-owning company's reporting system was not in line with company practices. As all safety outcomes are reports of unwanted incidents, we found it necessary to perform an analysis of the associations between the predictors and the total number of safety outcomes independent of post-categorizations. Further, in order to capitalize on the dataset to answer the pre-registered research question, other test of the hypotheses should be conducted before concluding with a null finding.

To compensate for the uncertainty in how events were coded and that an arbitrary division of events may hide associations, we modified the pre-registered model to an exploratory model where both event types were collapsed into one observed outcome variable. The explorative model showed a negative association between "Safe behaviour" and the combined safety outcomes. This indicates that, in line with the $\mathrm{H} 4$ expectation, the crew members that stated that they work safely had fewer accidents and reports of unwanted incidents on their vessels in the subsequent year. Such a mechanism requires that (1) crew members can assess and report their safety behaviour, (2) the behaviour has an impact on the safety of the vessel, and (3) the ship-owning company's KPI's are indicators of the level of safety on the vessels. There are few comparable studies in the literature on maritime safety, but Zohar (2000) found an association between safety climate and micro-accidents registered in the infirmary in a period of five months subsequent to the measures of selfreport in a metal processing plant and suggested that safety research should be designed to enable prediction of accidents.

4.2.2: Limitations with the survey measure. The other predictors, "SA", "Safety attitude" and "Reporting attitude", were not associated with safety outcomes. As discussed above, previous research has associated SA for a given task with performance on that task (see e.g. Endsley, 2019). It is therefore reasonable to assume that crew members who report that individual and environmental factors allow them to perceive relevant safety elements (level 1 of $\mathrm{SA}$ ), understand how they are connected (level 2 of SA), and anticipate what the elements and current situation could lead to (level 3 of SA) also perform their work more safely. If a context-general self-report is related to aspects of SA and determines how safely the crew are able to work, one may expect it to predict better safety outcomes for the vessel. However, limitations with the survey design may have obscured relationships between variables. The current inventory of SA measures the participants' assessment of their SA across work settings. As opposed to most SA measures, the current approach is thus not related to a specific task with clear evaluation criteria, but rather to an assessment of how participants think they handle safety in their work in general. Such an approach may be biased by the participants' knowledge, motivation and memory.

We expected that individuals who stated that they have positive attitudes to safety management and to the reporting system, would also work more safely. Previous research has shown that attitudes are related to behaviour (see e.g. Ajzen, 1991), and attitudes have been shown to partially mediate the relationship between safety climate and safety behaviour (Clarke, 2010). Relationships between attitudes and behaviour may hold in particular when there are specific attitudes related to specific actions (Ajzen \& Fishbein, 1973). This may hold better for reporting attitudes than for safety attitudes, as deciding whether to report a given event or not has a close correspondence to an attitude, while safety attitudes may correspond to several different decisions or actions. As mentioned above, social desirability bias may have contributed to obscure a relationship between attitudes and subsequent safety outcomes in the current study. When using self-report measurement of how the crew prioritizes safety, there may be particular biases to conform to the company's expected safety standard. However, the items in question do not have particularly sensitive content, and this could attenuate the impact of biases such as social desirability. Despite collaborating with the ship-owning company's personnel department to adapt the survey to the crew's assumed understanding of English as a second language, language issues may still have contributed to an inaccurate measure of attitudes. A prior inspection of items in the current dataset (Hjellvik et al., 2019, preprint) revealed that 
the negatively phrased items had lower average scores than the positively phrased items, which may indicate that the items were not always correctly interpreted. Such factors may have prevented real relationships from being identified.

4.2.3: Limitations with the safety outcomes. Failure to associate the survey measures to the safety outcomes may also be due to the company's KPIs not being reliable measures of actual safety. The industry has implemented such measures under the assumption that reports may provide reliable information about the general level of safety in an organization. Reports of incidents are assumed to provide knowledge about the level of safety in the workplace, used to monitor the frequency of undesirable events and to decide how to manage safety (Reiman \& Pietikäinen, 2012). However, as KPIs are reported by the crew and management, various factors may influence how well individuals are able and motivated to notice, reflect and report an incident accurately. ${ }^{2}$ Some organizations set targets for the number of reports the workforce is expected to submit. This may lead to a focus on managing the measure (Hopkins, 2009), and the crew may become preoccupied on "reporting for the sake of reporting" which may obscure a relationship between the number of reports and the actual level of safety. Macrae (2016) highlighted several problems with reporting systems in health care, and stated that reports may be biased by cognitive, social and organizational factors. There are for instance various reasons that could increase reports of some incidents and decrease reports of other, and this error rate would thus not reflect the actual level of safety. The KPIs used in the current study were a combination of the crew's voluntary reporting of accidents, "near-misses" and undesirable situations. KPIs of failure rates are more directly measured, and may be expected to be more honestly reported, as it would take a direct act of deception for employees to understate them (Hopkins, 2009). This could increase the reliability of such reports, as it is reasonable to assume that employees are honest and willing to maintain safety in their work. If the safety outcomes are not a reliable measure of the actual level of safety on the vessels, they would not be expected to be associated with safety determinants. However, the current study used the industry standard for monitoring safety, and such measures form the basis of most safety management systems.

\subsection{4: Limitations with the concept of reporting.}

As discussed in the Introduction, reporting may have a complex relationship to actual safety, which makes it challenging to predict the direction of the hypotheses. An increase in the reports of accidents or near-misses could indicate that a decrease in safety has led to more dangerous situations, but it could also indicate that a more safetyconscious crew are more able to recognize risks and motivated to report them. It is possible that there were effects in both directions, and that they cancelled out, or confounded any systematic variation between the variables in the current

${ }^{2}$ We hypothesized that "near-miss" reports would be more influenced by attitudes while events would be more influenced by behaviour, and that it could be informative to examine the relationship between the two types of KPIs. However, since none study. Vessel or company management may encourage the vessels to submit a minimum number of reports,

and it is difficult to know how such encouragement will be interpreted by the crew, and how it affects the reliability of the reports.

\section{3: Implications and Further research}

The literature review above has shown that various individual and organizational factors are associated with selfreported safety behaviour. The current study shows that selfreported safety behaviour predicts subsequent safety outcomes as individuals that work safely have fewer "near misses" and incidents. This indicates that large-scale surveys where employees report their safety-behaviour may be valuable in safety monitoring, and that safety management systems should take organizational, individual, interpersonal and socio-technical factors into consideration through multidisciplinary top-down system approaches (Rasmussen, 1997; Yemao, Monica, \& Scott, 2018). While there will always be variability in human performance, a safety management system that results in employees that are motivated, willing and able to work safely will facilitate beneficial safety outcomes.

While we found support for a relationship between subjective and objective measures when applying an exploratory SEM model, it should be noted that our preregistered model which distinguished between events and "near-misses" did not. This emphasizes how tenuous relationships may be in safety research, and the importance of having pre-registered hypotheses and analysis methods. The exploration of data should be clearly delineated from the confirmatory analyses in order to identify relationships that can be reliably reproduced in order to form the basis for safety management policies.

Further studies should investigate associations between self-reported safety factors and actual unwanted incidents. The relationship between reporting and accidents needs further exploration, and while this issue is complicated to resolve, it is no less important, since so much safety management research and practice relies on the assumed relationship between reporting and safety. We encourage further research on this issue, using reporting attitude measures supplemented by other approaches. A possible approach could be to present brief descriptions of hypothetical low risk situations and ask employees how likely it is that they would report such a case. It could also be beneficial to investigate various other measures of attitudes and cognitive states with actual safety behaviour. Further studies could benefit from including scales that measure biases such as social desirability along with measures of subjective and objective data. In addition, a longitudinal design could investigate predictors and outcomes over time. Such designs may provide more reliable information about

of the associations were significant, and we lost faith in how meaningful the division between the KPIs were, it is difficult to draw any conclusions on this matter. 
causal relationships, and safety interventions derived from safety research may thus have a stronger foundation.

\section{5: Conclusions}

Reliable empirical knowledge about causal relationships is needed to maintain safety in the maritime industry. The current study builds on the overall assumption that cognition, attitudes and behaviour predict subsequent safety outcomes on maritime vessels. The results showed an association between self-report of safety behaviour and objective safety outcomes, but there were no associations between the crew's SA and safety attitudes and objective safety outcomes. While there may be several reasons for null-findings, one should also allow for the possibility that there is no clear association between self-reported individual factors and safety. The variables measured are often mentioned as "safety critical", but the current study highlights the importance of designing studies where we can be more certain of the reliability and validity of results. A substantial part of safety research literature consists of associations between self-reported measures, either cross-sectional or longitudinally, and the majority of this research does not have a pre-registered transparent process with objective outcomes. Without establishing the external validity of the measures and the relationship to actual safety outcomes, and without being able to control for undeclared exploration of data, it is difficult to say how much confidence we should have in such findings. The current study thus suggests that some assumptions in past safety research may not hold true, and future research should address these issues.

\section{Acknowledgements}

We appreciate the assistance from the Utkilen ship-owning company and the safety managers Roy Hare and Haakon Losnegaard in particular. Sigurd W. Hystad assisted in the analysis work and Randi Elisabeth Hope Aga assisted in the survey development and collection of subjective data.

\section{References}

Ajzen, I. (1991). The theory of planned behavior. Organizational Behavior and Human Decision Processes, 50(2), 179-211. doi:10.1016/0749-5978(91)90020-T

Ajzen, I., \& Fishbein, M. (1973). Attitudinal and normative variables as predictors of specific behavior. Journal of Personality and Social Psychology, 27(1), 41-57. doi:10.1037/h0034440

Akyuz, E., \& Celik, M. (2015). A methodological extension to human reliability analysis for cargo tank cleaning operation on board chemical tanker ships. Safety Science, 75, 146-155. doi:10.1016/j.ssci.2015.02.008

Arslan, O., \& Er, I. D. (2008). SWOT analysis for safer carriage of bulk liquid chemicals in tankers. Journal of Hazardous Materials, 154(1-3), 901-913. doi:10.1016/j.jhazmat.2007.10.113

Celik, M. (2010). Enhancement of occupational health and safety requirements in chemical tanker operations: The case of cargo explosion. Safety Science, 48(2), 195-203. doi:10.1016/j.ssci.2009.08.004

Celik, M., \& Cebi, S. (2009). Analytical HFACS for investigating human errors in shipping accidents. Accident Analysis \& Prevention, 41(1), 66-75. doi:10.1016/j.aap.2008.09.004

Chauvin, C. (2011). Human Factors and Maritime Safety. Journal of Navigation, 64(4), 625-632. doi:10.1017/S0373463311000142

Chauvin, C., Lardjane, S., Morel, G., Clostermann, J.-P., \& Langard, B. (2013). Human and organisational factors in maritime accidents: Analysis of collisions at sea using the HFACS. Accident Analysis and Prevention, 59, 2637.

Clarke, S. (2010). An integrative model of safety climate: Linking psychological climate and work attitudes to individual safety outcomes using metaanalysis. Journal of Occupational and Organizational Psychology, 83(3), 553-578. doi:10.1348/096317909X452122
Cox, S., \& Cox, T. (1991). The structure of employee attitudes to safety: A European example. Work \& Stress, 5(2), 93-106. doi:10.1080/02678379108257007

Crano, W. D., \& Prislin, R. (2006). Attitudes and Persuasion. Annual Review of Psychology, 57(1), 345-374. doi:10.1146/annurev.psych.57.102904.190034

Dekker, S., \& Hollnagel, E. (2004). Human factors and folk models. Cognition, Technology \& Work, 6(2), 79-86. doi:10.1007/s10111-003-0136-9

Dekker, S., \& Nyce, J. (2012). Cognitive engineering and the moral theology and witchcraft of cause. Cognition, Technology \& Work, 14(3), 207-212. doi:10.1007/s10111-011-0203-6

Dekker, S. W. A., Hummerdal, D. H., \& Smith, K. (2010). Situation awareness: some remaining questions. Theoretical Issues in Ergonomics Science, 11(1-2), 131135. doi:10.1080/14639220903010092

Ding, S., Han, D. F., Li, X., \& Deng, W. (2014). Research of Human Errors in Maritime System. Applied Mechanics and Materials, 543, 4194-4197. doi:10.4028/www.scientific.net/AMM.543-547.4194

Donald, I., \& Canter, D. (1994). Employee attitudes and safety in the chemical industry. Journal of Loss Prevention in the Process Industries, 7(3), 203-208. doi:10.1016/0950-4230(94)80067-7

Edwards, A. L. (1953). The relationship between the judged desirability of a trait and the probability that the trait will be endorsed. Journal of Applied Psychology, 37(2), 90-93. doi:10.1037/h0058073

Endsley, M. R. (1995a). Measurement of Situation Awareness in Dynamic Systems. Human Factors: The Journal of Human Factors and Ergonomics Society, 37(1), 65-84. doi:10.1518/001872095779049499

Endsley, M. R. (1995b). Toward a Theory of Situation Awareness in Dynamic Systems. Human Factors: The Journal of Human Factors and Ergonomics Society, 37(1), 32-64. doi:10.1518/001872095779049543

Endsley, M. R. (2019). A Systematic Review and Meta-Analysis of Direct Objective Measures of Situation Awareness: A Comparison of SAGAT and SPAM. Human factors, 1-27. doi:10.1177/0018720819875376

Fenstad, J., Dahl, Ø., \& Kongsvik, T. (2016). Shipboard safety: exploring organizational and regulatory factors. Maritime Policy \& Management, 43(5), 552-568. doi:10.1080/03088839.2016.1154993

Hetherington, C., Flin, R., \& Mearns, K. (2006). Safety in shipping: The human element. Journal of Safety Research, 37(4), 401-411. doi:10.1016/j.jsr.2006.04.007

Hjellvik, L. R., Aga, R. E. H., \& Sxtrevik, B. (2019). Organizational and Individual Safety Factors in Work on Chemical Tanker Vessels [preprint]. Retrieved from https://doi.org/10.31234/osf.io/szr3f

Hofmann, D. A., \& Stetzer, A. (1996). A cross-level investigation of factors influencing unsafe behaviors and accidents. Personnel Psychology, 49(2), 307-339.

Hopkins, A. (2009). Thinking About Process Safety Indicators. Safety Science, 47(4), 460-465. doi:10.1016/j.ssci.2007.12.006

Hu, L.-T., \& Bentler, P. M. (1999). Cutoff criteria for fit indexes in covariance structure analysis: Conventional criteria versus new alternatives. Structural Equation Modeling: A Multidisciplinary Journal, 6(1), 1-55. doi:10.1080/10705519909540118

Lu, C.-S., \& Tsai, C.-L. (2010). The effect of safety climate on seafarers' safety behaviors in container shipping. Accident Analysis \& Prevention, 42(6), 1999-2006. doi:10.1016/j.aap.2010.06.008

Macrae, C. (2016). The problem with incident reporting. BMJ Quality \& Safety, 25(2), $71-75$

Nielsen, M. B., Eid, J., Hystad, S., Sætrevik, B., \& Saus, E. (2013). A brief safety climate inventory for petro-maritime organizations. Safety Science, 58, 81 88. doi:10.1016/j.ssci.2013.04.002

Nævestad, T.-O., Phillips, R. O., Størkersen, K. V., Laiou, A., \& Yannis, G. (2019). Safety culture in maritime transport in Norway and Greece: Exploring national, sectorial and organizational influences on unsafe behaviour and work accidents. Marine Policy, 99, 1-13. doi:10.1016/j.marpol.2018.10.001

Pedregon, C. A., Farley, R. L., Davis, A., Wood, J. M., \& Clark, R. D. (2012). Social desirability, personality questionnaires, and the "better than average" effect. Personality and Individual Differences, 52(2), 213-217. doi:10.1016/j.paid.2011.10.022

Pfeiffer, Y., Manser, T., \& Wehner, T. (2010). Conceptualising barriers to incident reporting: a psychological framework. Quality and Safety in Health Care, 1 10. doi:10.1136/qshc.2008.030445

Prince, S. A., Adamo, K. B., Hamel, M. E., Hardt, J., Connor G. S., \& Tremblay, M. (2008). A comparison of direct versus self-report measures for assessing physical activity in adults: a systematic review. The international journal of behavioral nutrition and physical activity, 5(1), 56. doi:10.1186/1479-5868-556

Probst, T. M., \& Graso, M. (2013). Pressure to produce=pressure to reduce accident reporting? Accident Analysis \& Prevention, 59, 580-587. doi:10.1016/j.aap.2013.07.020

Rasmussen, J. (1997). Risk management in a dynamic society: a modelling problem. Safety Science, 27(2-3), 183-213. doi:10.1016/S0925-7535(97)00052-0

Rasmussen, J. (2003). The role of error in organizing behaviour. Quality and Safety in Health Care, 12(5), 377-383. doi:10.1136/qhc.12.5.377 
Reiman, T., \& Pietikäinen, E. (2012). Leading indicators of system safety Monitoring and driving the organizational safety potential. Safety Science, 50(10), 1993-2000. doi:10.1016/j.ssci.2011.07.015

Rundmo, T. (1994). Associations between safety and contingency measures and occupational accidents on offshore petroleum platforms. Scandinavian Journal of Work Environment \& Health, 20(2), 128-131.

Rundmo, T., \& Hale, A. R. (2003). Managers' attitudes towards safety and accident prevention. Safety Science, 41(7), 557-574. doi:10.1016/S09257535(01)00091-1

Salmon, P., Stanton, N., Walker, G., \& Green, D. (2006). Situation awareness measurement: A review of applicability for C4i environments. Applied Ergonomics, 37(2), 225-238. doi:10.1016/j.apergo.2005.02.001

Sandhåland, H., Oltedal, H. A., Hystad, S. W., \& Eid, J. (2017). Effects of leadership style and psychological job demands on situation awareness and the willingness to take a risk: A survey of selected offshore vessels. Safety Science, 93, 178-186. doi:10.1016/j.ssci.2016.12.004

Sneddon, A., Mearns, K., \& Flin, R. (2013). Stress, fatigue, situation awareness and safety in offshore drilling crews. Safety Science, 56, 80-88. doi:10.1016/j.ssci.2012.05.027

Storgård, J., Erdogan, I., Lappalainen, J., \& Tapaninen, U. (2012). Developing Incident and Near Miss Reporting in the Maritime Industry-A Case Study on the Baltic Sea. Procedia - Social and Behavioral Sciences, 48(C), 10101021. doi:10.1016/j.sbspro.2012.06.1078

Strybel, T. Z., Vu, K.-P. L., Kraft, J., \& Minakata, K. (2008). Assessing the Situation Awareness of Pilots Engaged in Self Spacing. Proceedings of the Human Factors and Ergonomics Society Annual Meeting, 52(1), 11-15. doi:10.1177/154193120805200104

Sætrevik, B. (2013). Developing a context-general self-report approach to measuring three-level situation awareness. 66-71.

Sætrevik, B., \& Hjellvik, L. R. (2017). Reporting attitudes and determinants of incident reporting on maritime vessels [pre-registration]. Retrieved from osf.io/zGgdq

Sætrevik, B., \& Hystad, S. W. (2017). Situation awareness as a determinant for unsafe actions and subjective risk assessment on offshore attendant vessels. Safety Science, 93, 214-221. doi:10.1016/j.ssci.2016.12.012

Sætrevik, B., \& Hystad, S. W. (2019). Ship leadership, situation awareness and crew safety behaviour - Pre-registered replications in two survey datasets [preprint]. Retrieved from https://doi.org/10.31219/osf.io/y6jsa

Van 'T Veer, A. E., \& Giner-Sorolla, R. (2016). Pre-registration in social psychologyA discussion and suggested template. Journal of Experimental Social Psychology, 67, 2-12. doi:10.1016/jjesp.2016.03.004

van de Mortel, T. (2008). Faking it: social desirability response bias in self-report research. Australian Journal of Advanced Nursing, 25(4), 40-48.

Yemao, M., Monica, L., \& Scott, M. (2018). Towards a Pluralistic Epistemology: Understanding Human-Technology Interactions in Shipping from Psychological, Sociological and Ecological Perspectives. TransNav: International Journal on Marine Navigation and Safety of Sea Transportation, 12(4), 795-811. doi:10.12716/1001.12.04.20

Zohar, D. (2000). A Group-Level Model of Safety Climate: Testing the Effect of Group Climate on Microaccidents in Manufacturing Jobs. Journal of Applied Psychology, 85(4), 587-596. doi:10.1037/0021-9010.85.4.587 